\title{
The multifrequency campaign on 3C 279 in January 2006
}

\author{
W. Collmar ${ }^{1}$, M. Böttcher ${ }^{2}$, T. P. Krichbaum ${ }^{3}$, I. Agudo ${ }^{4,5,3}$, E. Bottacini ${ }^{1}$, M. Bremer ${ }^{6}$, V. Burwitz ${ }^{1}$, \\ A. Cuccchiara ${ }^{7}$, D. Grupe ${ }^{7}$, and M. Gurwell ${ }^{8}$ \\ 1 Max-Planck-Institut für extraterrestrische Physik, Giessenbachstrasse, 85748 Garching, Germany \\ e-mail: wec@mpe.mpg.de \\ 2 Astrophysical Institute, Department of Physics and Astronomy, Ohio University, Athens, OH 45701, USA \\ 3 Max-Planck-Institut für Radioastronomie, Auf dem Hügel 69, 53121 Bonn, Germany \\ 4 Institute for Astrophysical Research, Boston University, 725 Commonwealth Avenue, Boston, MA 02215, USA \\ 5 Instituto de Astrofísica de Andalucía (CSIC), Apartado 3004, 18080 Granada, Spain \\ ${ }^{6}$ IRAM, Avenida Divina Pastora 7, Local 20, 18012 Granada, Spain \\ 7 Pennsylvania State University, 525 Davey Lab, University Park, PA 16802, USA \\ 8 Harvard-Smithsonian Center for Astrophysics, 60 Garden Street, Cambridge, MA 02138, USA
}

Received 19 April 2010 / Accepted 12 July 2010

\section{ABSTRACT}

\begin{abstract}
Context. The prominent blazar 3C 279 is known for its large-amplitude variability throughout the electromagnetic spectrum and its often $\gamma$-ray-dominated spectral energy distribution. However, the characterization of its broadband spectral variability still lacks a consistent picture, and the origin of its high-energy emission is still unclear.

Aims. We intend to characterize the spectral energy distribution (SED) and spectral variability of 3C 279 in its optical high state.

Methods. Prompted by an optical high state of 3C 279, we organized an extensive multiwavelength campaign with coverage from radio to hard X-ray energies. The core components of the campaign were INTEGRAL and Chandra ToO observations in January 2006, augmented by X-ray data from Swift and RXTE as well as radio through optical coverage.

Results. The blazar was observed at a moderately high optical state. A well-covered multifrequency spectrum from radio to hard X-ray energies could be derived. During the flare, the radio spectrum was inverted, with a prominent spectral peak near $100 \mathrm{GHz}$, which propagated in time toward lower frequencies. The SED shows the typical two-bump shape, the signature of non-thermal emission from a relativistic jet. As a result of the long exposure times of INTEGRAL and Chandra, the high-energy spectrum $(0.3-100 \mathrm{keV})$ was precisely measured, showing - for the first time - a possible downward curvature. A comparison of this SED from 2006 to the one observed in 2003, also centered on an INTEGRAL observation, but during an optical low-state, revealed the surprising fact that despite a significant change of the high-frequency synchrotron emission (near-IR/optical/UV) - the low-energy end of the high-energy component (X-ray energies) remained virtually unchanged compared to 2003.

Conclusions. Our results prove that the two emission components do not vary simultaneously. This provides strong constraints on the modeling of the overall emission of 3C 279. When interpreted with a steady-state leptonic model, the variability among the SEDs displaying almost identical X-ray spectra at low flux levels, but drastically different IR/optical/UV fluxes, can be reproduced by a change solely of the low-energy cutoff of the relativistic electron spectrum. In an internal shock model for blazar emission, such a change could be achieved through a varying relative Lorentz factor of colliding shells producing internal shocks in the jet, and/or the efficiency of generating turbulent magnetic fields (e.g., through the Weibel instability) needed for efficient energy transfer from protons to electrons behind the shock.
\end{abstract}

Key words. galaxies: active - quasars: individual: 3C 279

\section{Introduction}

Blazars are the most variable and violent type of active galactic nuclei (AGN). They show rapid variability in all wavelength bands, polarized emission in the radio and optical bands, and often superluminal motion. The blazar subclass of AGN combines flat-spectrum radio quasars (FSRQs) and BL Lac objects. According to the unified model of AGN (e.g., Urry \& Padovani 1995), blazars are sources which expell jets close to our line of sight. The discovery by the experiments aboard the Compton Gamma-ray Observatory (CGRO) that blazars can radiate a large - sometimes even the major - fraction of their luminosity at $\gamma$-ray energies marked a milestone in our knowledge of blazars. During the CGRO mission about 90 blazars were detected by the different CGRO experiments at $\gamma$-ray energies, the majority by the Energetic Gamma-ray Experiment
Telescope (EGRET) at energies above $100 \mathrm{MeV}$ (Hartman et al. 1999). It is generally accepted that the radio to optical/UV continuum of blazars is synchrotron radiation generated by relativistic electrons in a magnetized jet. For the high-energy continuum from $\mathrm{X}$ - to $\gamma$-ray energies, two fundamentally different approaches are being considered (for a recent review see, e.g., Böttcher 2007). If protons are not accelerated to ultrahigh energies, exceeding the threshold for photo-pion production on the co-spatially produced synchrotron radiation field, high-energy emission might be dominated by Comptonization of soft photons by the non-thermal jet electrons (leptonic models; e.g., Maraschi et al. 1992; Dermer \& Schlickeiser 1993; Sikora et al. 1994). Otherwise, the high-energy radiative output might be dominated by proton-synchrotron radiation as well as synchrotron and Compton emission from the decay products of pions produced in photo-pion production processes (hadronic 
models; e.g., Mannheim \& Biermann 1992; Aharonian 2000; Mücke et al. 2003).

The optically violently variable (OVV) quasar 3C 279, located at a redshift of 0.536 , is one of the most prominent representatives of this source class. The blazar shows rapid variability in all wavelength bands, polarized emission in the radio and optical, superluminal motion, and a compact radio core with a flat radio spectrum. The quasar $3 \mathrm{C} 279$ was found to be a persistent - though highly variable - emitter of $\gamma$-rays above $100 \mathrm{MeV}$ by EGRET. Subsequently the source was frequently observed simultaneously at radio and optical bands, and was subject of many multiwavelength campaigns during the CGRO era (e.g., Maraschi et al. 1994; Hartman et al. 1996; Wehrle et al. 1998). A complete compilation of all simultaneous spectral energy distributions (SEDs) of 3C 279 collected during the lifetime of CGRO, including modeling of those SEDs with a leptonic jet model, is presented in Hartman et al. (2001a). The conclusion was that the high-energy emission most likely consists especially during flaring epochs - of several emission components that vary independently. The synchrotron self-Compton (SSC) mechanism (Maraschi et al. 1992; Bloom \& Marscher 1996) dominates the $\mathrm{X}$ - and soft $\gamma$-ray part while at higher $\gamma$-ray energies an external Compton component (e.g., Dermer \& Schlickeiser 1993; Sikora et al. 1994) becomes apparent.

Despite the enormous observational and theoretical multifrequency efforts, the nature and origin of the high-energy (X-rays to $\gamma$-rays) emission of $3 \mathrm{C} 279$ still remains unclear. Also, the connection of the high-energy radiation to the lowenergy radiation is not understood. Cross-correlations between different energy bands, particularly between optical, X-ray, and $\gamma$-rays were investigated by Hartman et al. (2001b), but no consistent trends could be found. This poses a serious challenge to the quite often adopted single-zone leptonic jet models, in which the same electron population produces both the low-frequency emission through synchrotron radiation and the high-energy emission through Compton scattering.

In order to improve on this general situation, Collmar et al. (2004) continued to observe 3C 279 in a multifrequency campaign organized around INTEGRAL (International Gamma-ray Astrophysics Laboratory) high-energy observations. INTEGRAL was launched in October 2002. In a campaign in June 2003, 3C 279 was detected significantly at hard X-ray energies for the first time by the INTEGRAL/IBIS experiment (Collmar et al. 2004). Those high-energy observations were supplemented in X-rays by a short Chandra pointing, and by ground based monitoring from radio to optical bands. In June 2003, the blazar was found in the faintest optical brightness (optical $R$-band: $\sim 17 \mathrm{mag}$ ) of the last 10 years, roughly 5 mag fainter than the maximum, and about 2.5 to 3 mag fainter than average. That campaign allowed Collmar et al. (2004) to compile a contemporaneous spectral energy distribution (SED) of an exceptional optical low-state.

In order to measure emission changes as function of optical brightness, we proposed for an intensive multifrequency campaign during an optical high state including high-energy INTEGRAL observations. On 5 January 2006, the trigger criterion was met when $3 \mathrm{C} 279$ exceeded an $R$-band flux corresponding to $R=14.5 \mathrm{mag}$. INTEGRAL observations were carried out together with supplementing multifrequency observations from radio via IR and optical to X-ray energies. Preliminary results from this campaign were reported by Collmar et al. (2007). Intensive ground-based monitoring in the IR and optical was assured by a WEBT (Whole Earth Blazar Telescope) campaign (see, e.g., Raiteri et al. 2006; Villata et al. 2007, and references therein). The results of the WEBT component of this campaign on 3C 279 in 2006 were presented in detail by Böttcher et al. (2007). In this paper we present details of the non-WEBT results of the radio, the X-ray, and soft $\gamma$-ray bands (Sect. 2), the interpretation of the radio data (Sect. 3), as well as the final SED (Sect. 4). In addition, we present a model interpretation of the $\mathrm{SED}$, and discuss our results in the framework of leptonic emission scenarios (Sect. 5).

Shortly after the core period of the multiwavelength campaign described here, the Major Atmospheric Gamma-ray Imaging Cherenkov Telescope (MAGIC) reported the detection of very-high-energy (VHE: $\gtrsim 200 \mathrm{GeV}$ ) $\gamma$-ray emission from 3C 279 on 23 February 2006 (Albert et al. 2008). This detection was extremely surprising for two reasons: (1) FSRQs like 3C 279 are known to have the peaks of their broad emission components in their SEDs at relatively low energies. In particular, the $\gamma$-ray component of 3C 279 has been known from the EGRET era to peak at a few $\mathrm{GeV}$, and the $\gamma$-ray spectrum was generally not expected to extend out to VHE frequencies at a substantial flux level. (2) 3C 279 is the VHE $\gamma$-ray source with the highest confirmed redshift to date. At such large redshifts, $\gamma \gamma$ absorption of VHE photons by the extragalactic background light (e.g., Dwek \& Krennrich 2005) is expected to lead to severe attenuation of the spectrum in the VHE regime. This prompted Albert et al. (2008) to propose that the universe may be more transparent to VHE $\gamma$-rays than previously thought (however, Stecker \& Scully 2009 dispute this claim). It has been shown by Böttcher et al. (2009) that if this VHE detection of 3C 279 can be confirmed, it would pose severe problems for any single-zone leptonic model interpretation and may potentially favor hadronic over leptonic models.

However, the reported VHE $\gamma$-ray flux, corrected for intergalactic $\gamma \gamma$ absorption, lies substantially above a straight extrapolation of non-simultaneous $\mathrm{MeV}-\mathrm{GeV}$ spectra measured by EGRET as well as more recently by the Fermi Gamma-ray Space Telescope (Abdo et al. 2009). Also, in spite of intensive efforts, no other VHE $\gamma$-ray observatory has so far been able to confirm the detection of VHE $\gamma$-ray emission from 3C 279. This indicates that the detection of 23 February 2006 must correspond to an extraordinary flaring activity and cannot be considered representative of the time of the simultaneous multiwavelength observations reported here. Therefore, we will focus our modeling efforts on leptonic models, which have been shown to provide a successful description of all multiwavelength SEDs of 3C 279 throughout the CGRO era (Hartman et al. 2001a).

For the purpose of the model interpretation, we will use the widely accepted $\Lambda \mathrm{CDM}$ cosmology with $H_{0}=$ $70 \mathrm{~km} \mathrm{~s}^{-1} \mathrm{Mpc}^{-1}, \Omega_{\mathrm{m}}=0.3$, and $\Omega_{\Lambda}=0.7$. In this cosmology, the luminosity distance of $3 \mathrm{C} 279$ is $D_{\mathrm{L}}=3.08 \mathrm{Gpc}$. Throughout the paper we refer to the energy spectral index $\alpha$, defined through $F_{v}[\mathrm{Jy}] \propto v^{-\alpha}$, and the photon spectral index $\Gamma_{\mathrm{ph}}=\alpha+1$.

\section{Observations, data reduction, and results}

After 3C 279 met the trigger criterion for the targeted optical high-state $(R \leq 14.5 \mathrm{mag})$ on 5 January 2006, we requested the pre-approved high-energy ToO observations with INTEGRAL and Chandra. To monitor the X-ray and UV-state of 3C 279 during the INTEGRAL observations, we proposed and were granted monitoring observations by Swift. In addition, ground-based monitoring observations in the framework of a WEBT campaign as well as sub-millimeter and radio monitoring of several telescopes were contributed to our campaign. In this section, we describe details of the collection and analysis of the previously 
Table 1. Observation log of the high-energy observations during our January 2006 campaign. For INTEGRAL the orbital revolution number is given.

\begin{tabular}{lcccr}
\hline \hline Obs. Periods & T-Start & T-Stop & Exposure & Satellite \\
\hline 001 & $2006-01-1300: 40$ & $2006-01-1323: 37$ & 15014 & Swift \\
002 & $2006-01-1400: 42$ & $2006-01-1502: 35$ & 10613 & \\
003 & $2006-01-1604: 07$ & $2006-01-1623: 34$ & 1581 & \\
004 & $2006-01-1701: 03$ & $2006-01-1723: 53$ & 19289 & \\
005 & $2006-01-1801: 14$ & $2006-01-1824: 00$ & 13897 & \\
006 & $2006-01-1901: 08$ & $2006-01-1906: 14$ & 4113 & \\
\hline 001 & $2006-01-1708: 59$ & $2006-01-1717: 51$ & 31920 & Chandra \\
\hline 397 & $2006-01-1300: 22$ & $2006-01-1503: 03$ & 174879 & INTEGRAL \\
$398 / 399$ & $2006-01-1607: 17$ & $2006-01-2017: 41$ & 322326 & \\
400 & $2006-01-2205: 13$ & $2006-01-2209: 25$ & 14392 & \\
\hline
\end{tabular}

Notes. ${ }^{(a)}$ Start and end times are given in UT; ${ }^{(b)}$ exposure is given in s.

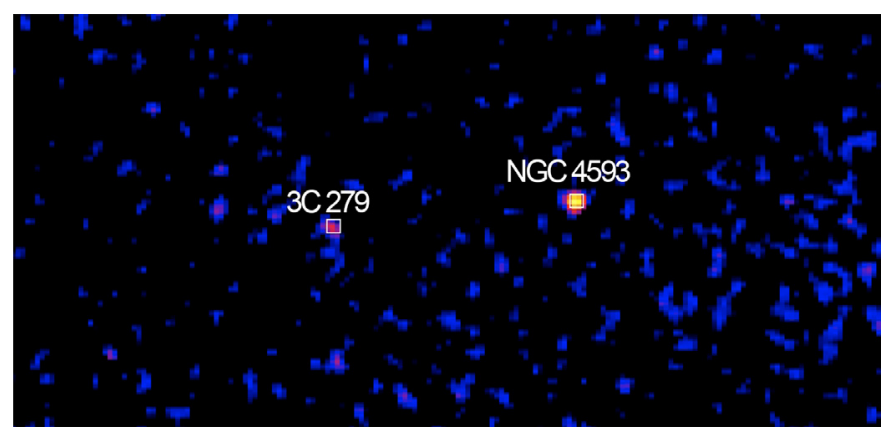

Fig. 1. The ISGRI image shows a $6.4 \sigma$ detection of 3C 279 in the $20-60 \mathrm{keV}$ band. In addition, the Seyfert galaxy NGC 4593 is even more clearly detected. The image shown covers a sky region of $\sim 11.8^{\circ} \times 5.6^{\circ}$.

unpublished data and briefly summarize the relevant aspects of campaign contributions which were previously published elsewhere (Böttcher et al. 2007, 2009).

\subsection{INTEGRAL}

INTEGRAL is an ESA scientific mission dedicated to highresolution spectroscopy with SPI (Vedrenne et al. 2003) and imaging with IBIS/ISGRI (angular resolution: 12' FWHM, point source location accuracy: $\simeq 1^{\prime}-3^{\prime}$; IBIS/ISGRI, see Ubertini et al. 2003; Lebrun et al. 2003) of celestial sources in hard Xand soft $\gamma$-rays $(15 \mathrm{keV}$ to $10 \mathrm{MeV})$. In addition, INTEGRAL provides simultaneous monitoring at X-rays $(3-35 \mathrm{keV})$ with JEM-X (Lund et al. 2003) and at optical wavelengths (Johnson $V$-filter) with OMC (Mas-Hesse et al. 2003).

INTEGRAL observed the blazar in three pointings between 13 January and 22 January 2006 for a total observation time of $\sim 512 \mathrm{ks}$ (Table 1). Due to annealing, no INTEGRAL SPI data are available. The INTEGRAL analyses were done with the INTEGRAL Offline Scientific Analysis (OSA) version 7.0.

The IBSI/ISGRI data were screened for data failures or problems, and in the end a total observation time of $505043 \mathrm{~s}$, available in 149 so-called science windows, was selected for final analysis. IBIS/ISGRI detected the blazar at energies between 20 and $100 \mathrm{keV}$ with a significance of $\sim 7.5 \sigma$ during the total used exposure of $505 \mathrm{ks}$. The ISGRI image (Fig. 1) of the $20-60 \mathrm{keV}$ band shows a $6.4 \sigma$ detection of $3 \mathrm{C} 279$. Other Virgo region

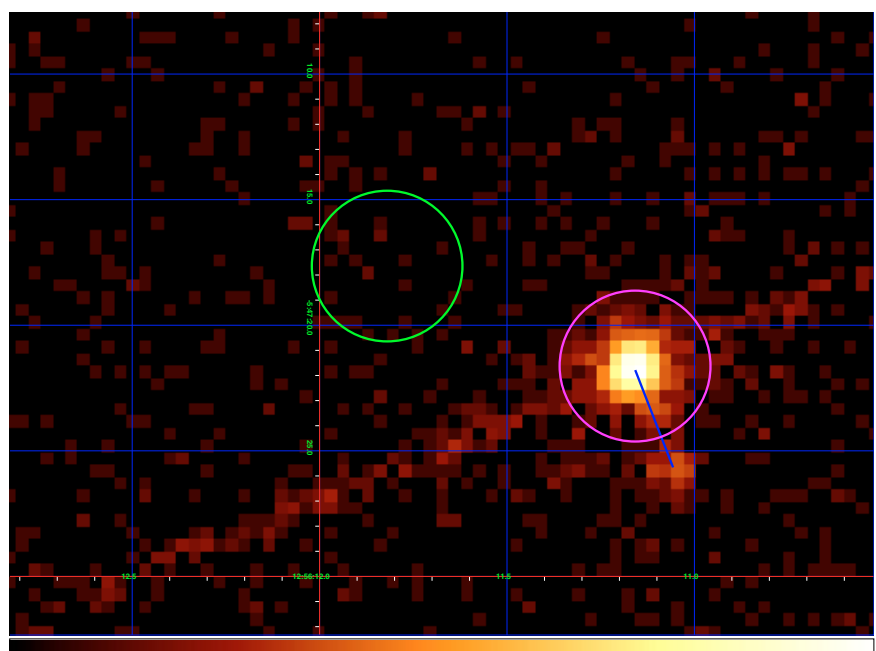

Fig. 2. The blazar is clearly visible in the Chandra image. A weaker source, probably the X-ray jet of 3C 279 , is also visible at an angular distance of $\sim 4$ arcsec. The purple (source) and the green (background) circles indicate the sky regions used for data analysis. The image shown covers a sky region of $\sim 34.0^{\prime \prime} \times 24.5^{\prime \prime}$.

sources are also detected in these observations, for example the prominent quasar 3C 273 and the Seyfert galaxy NGC 4593. The blazar was measured by ISGRI at a surprisingly low flux level of $(2.53 \pm 0.52) \times 10^{-4} \mathrm{ph} \mathrm{cm}^{-2} \mathrm{~s}^{-1}$. The spectral analysis with XSPEC between 20 and $100 \mathrm{keV}$, assuming a power-law shape, yields a weakly determined average spectral shape (Fig. 3) of the photon index $\Gamma_{\mathrm{ph}}=2.0 \pm 0.5(1 \sigma)$.

Subdividing the observations into three roughly equal time intervals (13-15 January, 16-18 January, and 18-20 January), yields a low significance flux value for each interval. No significant variability is seen, but there appears to be a trend of a rising hard X-ray flux (20-60 keV) toward the end of INTEGRAL's observational period (Fig. 4).

JEM-X did not detect 3C 279. Upper limits were derived for two energy bands (5-10 and 10-20 keV) based on the mosaic images from these observations. The OMC monitoring data were analyzed as well (see Bottacini et al. 2007). We do not include them in this paper, because during the campaign superior optical measurements were performed by several ground-based observatories (see Sect. 2.5) as well as the Swift UVOT (Sect. 2.3). 


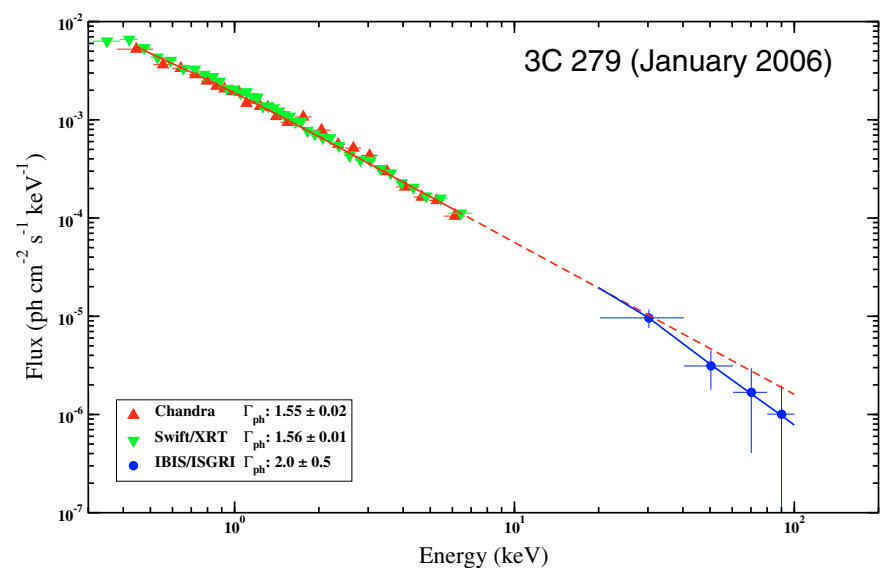

Fig. 3. Soft to hard X-ray spectrum of 3C 279 as measured by Chandra (17 January 2006; exposure $\sim 32 \mathrm{ks}$ ), Swift (6 pointings between 13-19 January 2006; exposure $\sim 65 \mathrm{ks}$ ) and INTEGRAL/ISGRI (3 pointings between 13-22 January 2006; exposure $\sim 505 \mathrm{ks}$ ) together with the Chandra (the Swift is completely consistent with Chandra) and ISGRI best-fit power-law shapes (solid lines). The dashed line shows the extrapolation of the Chandra spectrum up to $100 \mathrm{keV}$, indicating the softer ISGRI spectrum above $\sim 20 \mathrm{keV}$. The best-fitting photon indices including their errors $(1 \sigma)$ are given.

\subsection{Chandra}

After the INTEGRAL observations were approved, we also triggered our pre-approved Chandra $30 \mathrm{ks}$ ToO observation on 3C 279. It was scheduled on 17 January 2006, roughly centered in time on the one week INTEGRAL observation. Chandra observed the blazar for a total observation time of $\sim 32 \mathrm{ks}$ in order to determine contemporaneously and most accurately the X-ray state and spectrum of 3C 279. To avoid pile-up in the Chandra detectors of the assumed strong X-ray source, the LETG-ACIS-S mode was used.

The blazar 3C 279 was significantly detected by Chandra. The Chandra X-ray image (Fig. 2) shows next to it a weaker $\mathrm{X}$-ray source at a distance of $\sim 4$ arcsec. This could be emission from its jet because it is along the direction of its VLA jet (e.g. Akujor et al. 1994). Chandra measured a well determined $\mathrm{X}$-ray spectrum between 0.3 and $7 \mathrm{keV}$ (Fig. 3). Assuming the canonical power-law shape at X-ray energies, a spectral photon index of $\Gamma_{\mathrm{ph}}=1.55 \pm 0.02$ was derived. The spectrum is consistent (reduced $\chi^{2}: 1.14$ ) with a hard power-law shape. However, the spectral analysis indicated a spectral bending from a harder to a softer spectrum toward higher energies. A fit with a broken powerlaw shape yielded a better fit (reduced $\chi^{2}: 0.82$ ): a harder shape (photon index: $1.46 \pm 0.03$ ) below $\sim 3 \mathrm{keV}$ and softer one (photon index: $1.87 \pm 0.12$ ) above. In particular, no soft excess was found, which would have indicated a contribution of the synchrotron component or the accretion disk to the soft X-ray emission. Chandra did not observe any variability of 3C 279 during the uninterrupted source-pointing of $\sim 8 \mathrm{~h}$.

\subsection{Swift}

The $\gamma$-ray burst explorer Swift was launched in 2004, and carries three co-aligned detectors (Gehrels et al. 2004): the Burst Alert Telescope (BAT, Barthelmy et al. 2005), the X-ray Telescope (XRT, Burrows et al. 2005) and the Ultraviolet/Optical Telescope (UVOT, Roming et al. 2005). Between 13 and 19 January 2006, Swift regularly observed the blazar, and - by our ToO request - contemporaneously with

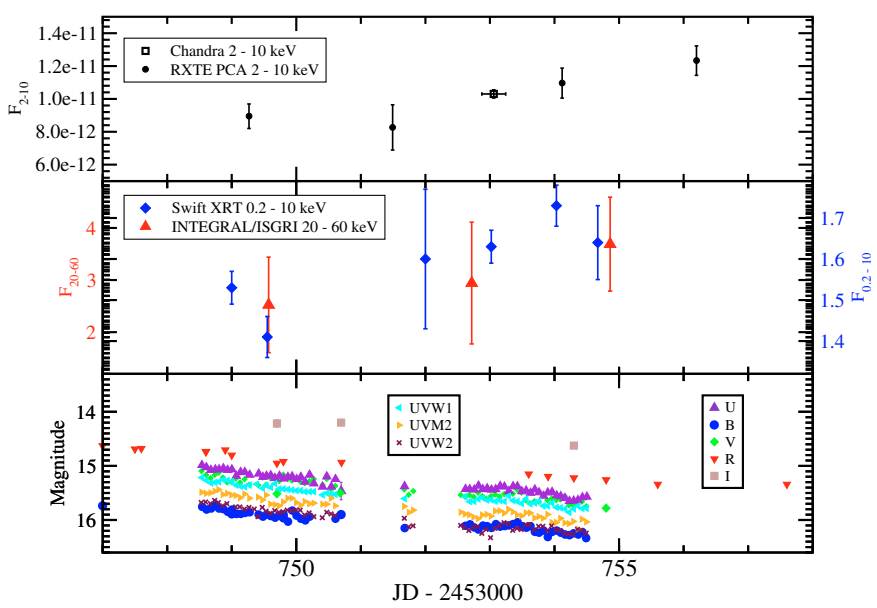

Fig. 4. X-ray (top two panels) and optical/UV (lower panel) light curves of 3C 279 during the core campaign period, 13-22 January 2006. Units for the X-ray light curves are: $F_{2-10}\left[\mathrm{erg} \mathrm{cm}^{-2} \mathrm{~s}^{-1}\right]$ and $F_{20-60}$ $\left[10^{-4} \mathrm{ph} \mathrm{cm}^{-2} \mathrm{~s}^{-1}\right]$.

INTEGRAL (Table 1). The data analysis followed the standard procedure for Swift XRT data (e.g. Grupe et al. 2010) by using the HEASOFT 6.8 tools and XSPEC 12.5.1.n (Arnaud 1996). All observations were performed in photon-counting mode (Hill 2004). Source photons were selected in the circular region with a radius of $82.5^{\prime \prime}$. Spectral data were re-binned to 50 counts per bin (except for segment 003 where we used 20 counts per bin).

The XRT measured a moderately variable $0.2-10 \mathrm{keV}$ X-ray flux with a variability amplitude of $\Delta F / F \lesssim 25 \%$. After an initial decline throughout 13 January 2006, the Swift XRT flux seems to indicate a gradual rising trend during the remainder of the INTEGRAL observation period, in agreement with the trend seen by RXTE in the $2-10 \mathrm{keV}$ range (see Fig. 4).

Parallel to XRT, UVOT monitored 3C 279's ultraviolet and optical emission. The UV magnitudes have been de-reddened using the extinction curves of Cardelli et al. (1989) with $R_{V}=3.1$ and $A_{V}=0.095$, taken from the NASA/IPAC Extragalactic Database $\left(\mathrm{NED}^{1}\right)$. The data indicate a continuous fading of the source in all six filter bands (Figs. 4 and 7), in agreement with the trend observed with ground-based optical observatories (see Sect. 2.5).

\subsection{RXTE}

In the course of a long-term monitoring program led by A. Marscher of $\gamma$-ray bright blazars using the Proportional Counter Array (PCA) on board the Rossi X-ray Timing Explorer (RXTE), 3C 279 was observed with 2-3 pointings per week throughout (and beyond) the campaign period. Details of the observations and data analysis have been published in Chatterjee et al. (2008), and the X-ray data have been discussed in the context of the WEBT campaign (see Sect. 2.5) and the MAGIC detection in Böttcher et al. (2009). The RXTE data are included in Fig. 4, and the multiwavelength light curves (radio through hard X-rays) throughout January and February 2006 are shown in Fig. 7.

Before and during the core period of our multiwavelength campaign, the RXTE light curve showed 3C 279 to be in a low flux state, near its historical minimum, with a moderately soft spectrum with $\alpha \sim 1$ in the $2-10 \mathrm{keV}$ range. Toward the end of January 2006, i.e., after the core campaign period, the object

1 http://nedwww.ipac.caltech.edu/ 
went into a high-activity $\mathrm{X}$-ray state with average X-ray flux a factor $\sim 2-3$ higher than during the previous low state. In this state, 3C 279 showed substantial, correlated flux and spectral variability on time scales of $\sim 10$ days. Notably, the time of the MAGIC detection occurred during the rising phase of a major $\mathrm{X}$-ray outburst, peaking about one week thereafter at a flux level of $\sim 5$ times the previous quiescent flux. In the RXTE X-ray band 3C 279 is continuously variable with flux variations up to a factor of $10\left(F_{2-10}\right.$ between $\sim 0.5$ and $\left.5 \times 10^{-11} \mathrm{erg} \mathrm{cm}^{-2} \mathrm{~s}^{-1}\right)$. See e.g. Marscher (2006) for a longterm (1996-2005) RXTE X-ray light curve of 3C 279.

\subsection{Optical monitoring}

The quasar 3C 279 is regularly monitored at optical, nearinfrared, and radio frequencies by a number of observatories associated with the Whole Earth Blazar Telescope (WEBT). It was the monitoring provided by the WEBT optical partners that led to the discovery of the high state of 3C 279 on 5 January 2006, which triggered our multiwavelength campaign. Early results of this campaign were published and discussed by Böttcher et al. (2007). Here we add additional observations in the radio-to-sub$\mathrm{mm}$ regime from radio observatories, which are not asscociated with the WEBT, and which complement the spectral and time coverage considerably. The combined radio, sub-millimeter, optical, and X-ray data are shown in Figs. 4-7.

In the optical, the WEBT monitoring of December 2005March 2006 indicated an overall elevated optical state, with variability amplitudes of $\sim 0.5 \mathrm{mag}$ on time scales of several days. The most remarkable light curve feature during that campaign was an unusually clean, quasi-exponential decay of the flux in all optical bands (BVRI) over a period of about two weeks ( JD 2 453 744-2 453 757), including the core period illustrated in Figs. 4 and 7, shortly after exceeding the trigger threshold of $R=14.5 \mathrm{mag}$. This decay could be well approximated by an exponential $F(t)=F_{0} \mathrm{e}^{-t / \tau}$ with a time scale of $\tau \sim$ 12.8 days, and continued throughout the time period of the highenergy (INTEGRAL, Chandra, Swift) observations. Böttcher \& Principe (2009) suggest that this clean, quasi-exponential decay may be the signature of deceleration of a ballistically moving plasmoid along the jet.

The light curve recovered to a high level right after the period of the high-energy observations (around 22 January) and 3C 279 remained optically bright until the end of March 2006. This includes the time of the MAGIC detection (23 February 2006), during which the optical light curve did not show any remarkable variability features. While Böttcher et al. (2007) found hints for spectral hysteresis and a hard lag within the optical bands in light curve segments later during the WEBT campaign, there was no evidence for optical spectral variability during the multiwavelength core campaign period (JD 2453748-2453758, i.e., 12-22 January 2006).

\subsection{Radio to sub-millimeter variability}

The blazar 3C 279 was observed in the radio- to sub-mm bands by a number of different observatories. The data sets are compiled from ongoing regular flux density monitoring programs and complemented by dedicated target-of-opportunity observations performed during 2005 and 2007. The total of all flux density measurements cover a frequency range from 1.4 to $345 \mathrm{GHz}$, with the following contributions: the $100 \mathrm{~m}$ Effelsberg telescope of the Max-Planck-Institut für Radioastronomie (Bonn,
Germany) observed at 1.4, 2.7, 5, 8.4, 10.5, 15, 22, 32 and $43 \mathrm{GHz}$, providing flux density measurements which are quasisimultaneous in time (for details see: Fuhrmann et al. 2008; Angelakis et al. 2008).

The IRAM $30 \mathrm{~m}$ telescope on Pico Veleta (Spain) contributed with flux density measurements at 90 and $230 \mathrm{GHz}$. These data resulted from the regular IRAM flux monitoring program (see Ungerechts et al. 1998; and Agudo et al. 2006, for a decription of the data reduction), and additional data obtained on the basis of target of opportunity observations proposed by us. These flux density measurements are complemented by additional measurements at 90 and $230 \mathrm{GHz}$ with the $6 \times 15 \mathrm{~m}$ IRAM interferometer on Plateau de Bure (France) and at $90 \mathrm{GHz}$ with the Australia Telescope Compact Array (ATCA). To fill some gaps in the time coverage, we also included data from the VLA polarization monitoring, which is performed at $5 \mathrm{GHz}$, $8.4 \mathrm{GHz}, 22$ and $43 \mathrm{GHz}$ (Taylor \& Myers 2000). We further included the $14.5 \mathrm{GHz}$ data from the University of Michigan Radio Observatory (UMRAO) monitoring program and the 230 and $345 \mathrm{GHz}$ data from the AGN monitoring of the Submillimeter Array (SMA) on Mauna Kea (Hawaii). The data from UMRAO and the SMA were already published in part by Chatterjee et al. (2008) and Larionov et al. (2008), where also the corresponding data reduction and calibration is described.

In Fig. 5 we plot the flux density of 3 C 279 in the radio bands versus time for the period 2004.4-2007.8. We have also added the optical $R$-band flux density from Chatterjee et al. (2008). In the lower panel of the figure we show the fluxdensity variability of the VLBI core component at $43 \mathrm{GHz}$ (same data as in Chatterjee et al. 2008, but fitted with a circular Gaussian beam). Furthermore we add the times of zero separation from the VLBI core for the jet components C22 $(2005.18 \pm 0.06), C 23(2006.415 \pm 0.15)$, and $(2006.894 \pm 0.11)$ for C24 (see Chatterjee et al. 2008; Larionov et al. 2008).

From Fig. 5 it is obvious that the variability amplitudes are more pronounced in the optical and mm-bands than in the cm-regime, which is consistent with a synchrotron selfabsorbed radio jet. Between 2005.9 and 2007.0 the optical to radio fluxes rose, indicative of an optical/radio flare, which peaked by the end of year 2007. During the rising phase of this flare, a first local flux density maximum at mm-wavelengths, respectively a plateau in the $14.5 \mathrm{GHz}$ data was reached around 2006.4-2006.5, about half a year before the absolute maximum of the flux densities were reached (peak: 2006.9-2007.0). This local maximum is clearly seen in the SMA data at $345 \mathrm{GHz}$ $(0.8 \mathrm{~mm})$ and $230 \mathrm{GHz}(1 \mathrm{~mm})$ and also in the VLBI core flux at $43 \mathrm{GHz}(7 \mathrm{~mm})$. This indicates the superposition and blending of two flares separated in time by about $0.4-0.5 \mathrm{yr}$. It is tempting to associate the ejection of the VLBI components C23 and C24 with the observed double-peak structure between 2006 and 2007 in the total flux densities and in the VLBI core flux. After 2007.0, the optical flux density decayed very fast, followed by successively more shallow decays between sub-mm and cm-wavelengths.

Between frequencies of $345 \mathrm{GHz}$ and $90 \mathrm{GHz}$, the variability is fast and the light curves are partially undersampled in time. With the present time sampling, the fastest significant variations appear on time scales of $\leq 20-40$ days. Faster variations cannot be excluded, but at this point remain speculative. At $90 \mathrm{GHz}$ a flux density increase from 12.8 in 2006.036 to 17.7 Jy in 2006.125 is observed in 34 days. This corresponds to a rate of $0.14 \mathrm{Jy} /$ day for the flux density scale. This in turn leads to an apparent brightness temperature of the variable component of $T_{\mathrm{B}} \geq 8.4 \times 10^{12} \mathrm{~K}$, which could be reduced to values 

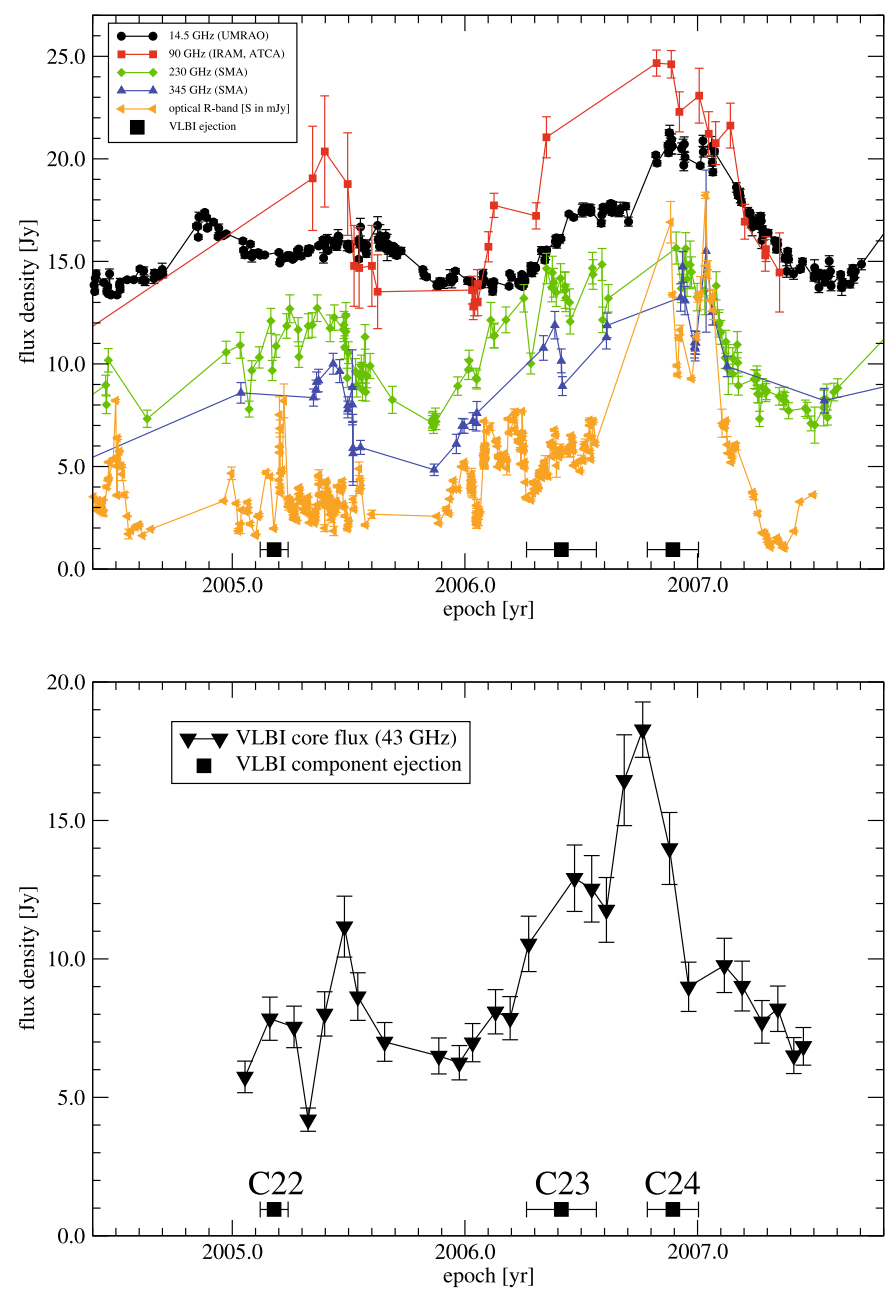

Fig. 5. Top: radio and optical flux density plotted versus time. Different symbols and colors denote different observing frequencies: $14.5 \mathrm{GHz}$ black circles, $90 \mathrm{GHz}$ red squares, $230 \mathrm{GHz}$ green diamonds, $345 \mathrm{GHz}$ blue triangles, and optical $R$-band (amplitudes given in [mJy]) yellow triangles. Black squares mark VLBI component ejection as indicated in the panel below. Bottom: $43 \mathrm{GHz}$ VLBI core flux density versus time. Black squares with horizontal error bars denote the linearly backextrapolated ejection times of the VLBI components C22, C23 and C24 (in order of their appearance).

below the inverse-Compton limit of $10^{12} \mathrm{~K}$, if the Doppler-factor is larger than $\delta \geq 4.4$ (adopting the equations given in Fuhrmann et al. 2008). With this Doppler-factor the corresponding linear dimension of the emission region would be $42 / \delta=9.5 \mu$ as, which corresponds to a linear scale of $r \leq 7.9 \times 10^{17} / \delta=1.8 \times$ $10^{17} \mathrm{~cm}$. Using the above Doppler factor and a typical speed of the VLBI components of $\beta_{\text {app }} \simeq 15 \mathrm{c}$, we obtain a minimum bulk Lorentz-factor of the jet of $\gamma_{\min } \geq 15$ and an inclination of the inner jet with respect to the line of sight at an angle of $i \leq 7^{\circ}$.

\subsection{Quasi-simultaneous radio spectra}

From the available radio data presented in the previous section, it is possible to construct quasi-simultaneous broad-band radio spectra. Because of the irregular time sampling at the different observatories, the binning of the data in time is different for each epoch and was chosen to obtain the best possible frequency coverage. In Fig. 6 we show some examples of the radio

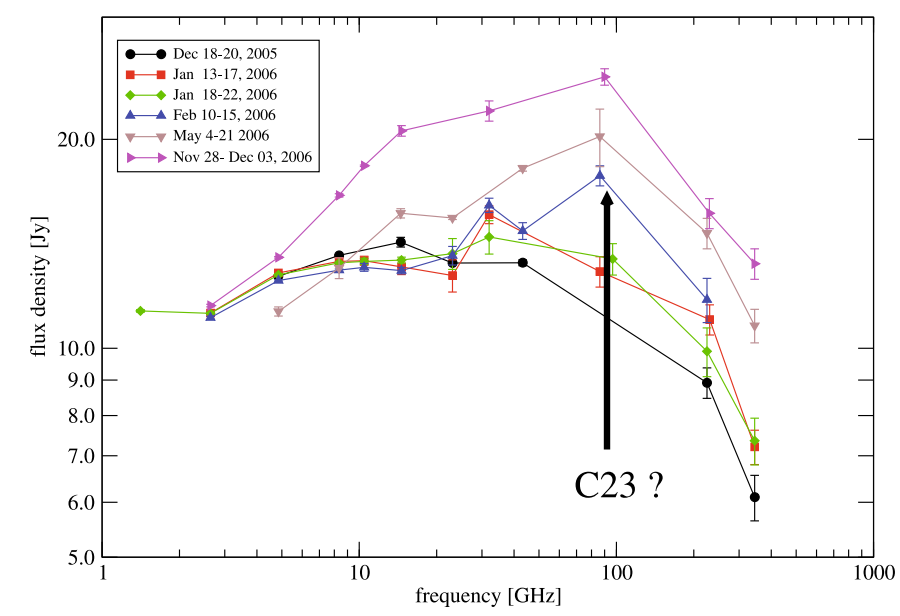

Fig. 6. Quasi-simultaneous broad-band radio spectra of 3C 279. Different symbols and colors denote different observing epochs, as indicated by the legend box in the top left of the figure. Because of the irregular time sampling at the different observatories, the binning intervals differ from epoch to epoch. The spectrum of November 2006 was observed near the time of the peak of the 2007.0 radio flare, see Fig. 5. The solid black arrow marks the spectral flaring component, which may be associated with the new VLBI jet components C23 in Larionov et al. (2008).

spectra, which cover the frequency range from 1.4 to $350 \mathrm{GHz}$. The legend at the top left of the figure shows the observing date and binning interval. The spectra are remarkably complex and show spectral evolution on time scales of weeks to months. The spectrum of December 2005 (circles) was observed just at the time before the 2006-2007 activity started and the 2007 flare began. The radio spectrum is flat up to about $43 \mathrm{GHz}$, and steepens toward shorter (sub-mm) wavelengths $\left(\alpha_{43 / 225 \mathrm{GHz}} \simeq 0.25\right.$, $\alpha_{225 / 345 \mathrm{GHz}} \simeq 0.9$ ). From January 2006 to May 2006, the spectrum becomes more and more inverted, with a prominent spectral maximum near $100 \mathrm{GHz}$. In the context of the three-stage shock in jet model of Marscher \& Gear (1985; see also Lindfors et al. 2006), this behavior can be interpreted as a spectral component, which is in its inverse-Compton stage (rising turnover flux $S_{m}$ and decreasing turnover frequency $v_{m}$ ). Between $10-100 \mathrm{GHz}$, the observed spectral slopes vary considerably in all epochs. This suggests another variable spectral component with lower turnover frequency, which is less prominent than the one discussed before. In February 2006, this spectral component is best visible, causing a spectral break near $43 \mathrm{GHz}$, and it may have propagated down to $15 \mathrm{GHz}$ by May 2006. Owing to the lack of multi-frequency VLBI data during this time, it is not possible to decompose the radio spectrum of the total flux into several spectra of distinct VLBI jet components. However, with regard to the published $43 \mathrm{GHz}$ VLBI maps and the known jet kinematics (Chatterjee et al. 2008; Larionov et al. 2008), it is very likely that the more prominent spectral break near $100 \mathrm{GHz}$ is associated with a new jet component, which must have emerged from the self-absorbed VLBI core region between mid-January and mid-February 2006. A likely candidate for an association of that spectral feature with a new jet component would be $\mathrm{C} 23$, which became visible at $43 \mathrm{GHz}$ after $t_{0}=2006.415 \pm 0.15$ (see solid black arrow in Fig. 6). We note that an association of a secondary and less pronounced spectral break in the $20-30 \mathrm{GHz}$ range with an evolving older jet component $(\mathrm{C} 22$ ejected at $\simeq 2005$.2) is a more speculative, but not unreasonable interpretation. 


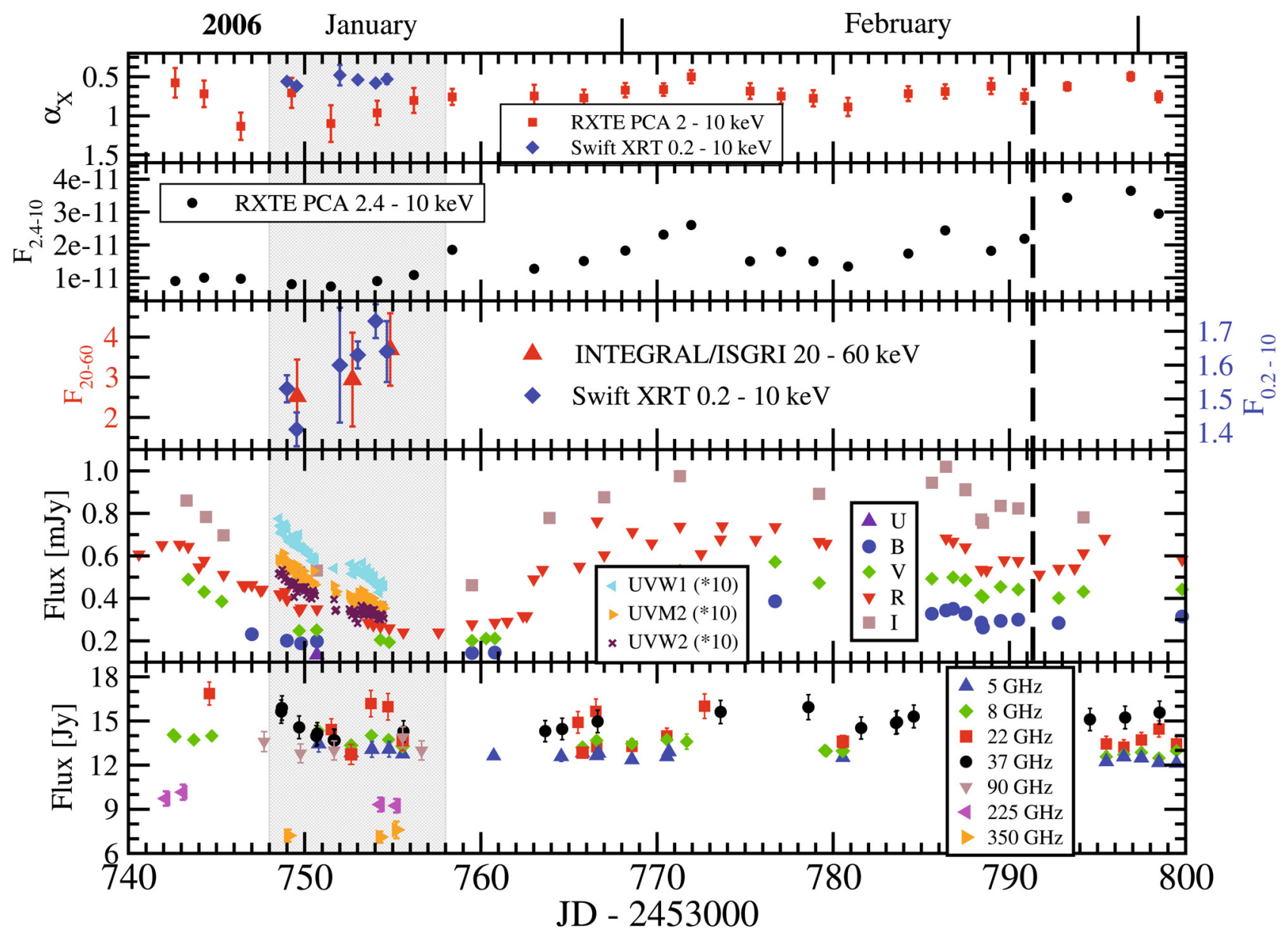

Fig. 7. Multiwavelength light curves of 3C 279 during January and February 2006. The gray-shaded area indicates the core campaign period of the INTEGRAL, Chandra, and Swift observations; the vertical dashed line indicates the time of the MAGIC VHE $\gamma$-ray detection. Units of the X-ray fluxes are: $F_{2-10}\left[\mathrm{erg} \mathrm{cm}^{-2} \mathrm{~s}^{-1}\right]$ and $F_{20-60}\left[10^{-4} \mathrm{ph} \mathrm{cm}^{-2} \mathrm{~s}^{-1}\right]$. The top panel shows the RXTE $(2-10 \mathrm{keV})$ and Swift/XRT $(0.2-10 \mathrm{keV})$ spectral indices. The RXTE data are from Chatterjee et al. (2008), updated by A. Marscher (private comm.).

\section{Interpretation of the radio data}

The close correlation of the VLBI core flux with the total flux (see Fig. 5, and Chatterjee et al. 2008; Larionov et al. 2008) allows us to attribute most of the variability to be located within the synchrotron self-absorbed VLBI core, for which an upper limit to its size $\theta$ is available $\left(\delta \geq \theta D_{\mathrm{L}} /\left(c t_{\mathrm{var}}\right) /(1+z)\right.$, with luminosity distance $D_{\mathrm{L}}$ and variability time scale $\left.t_{\mathrm{var}}\right): \theta \leq 0.03$ mas or $r \leq 5.7 \times 10^{17} \mathrm{~cm}$. Applying the light travel time argument and following Jorstad et al. (2005), a lower limit to the relativistic Doppler factor of $\delta \geq 5.1$ is obtained, which is close to the Doppler factors estimated in the previous sections. For synchrotron self-absorption the magnetic field can be calculated via $B_{\mathrm{ssa}}[\mathrm{G}] \propto 2.3 \times 10^{-5} \Delta S^{-2} v_{m}^{5} \theta^{4} \delta /(1+z)$. This leads to $B_{\mathrm{ssa}} \simeq 15 \mathrm{mG}$ for the VLBI core and inner jet, where we adopted $v_{m}=90 \mathrm{GHz}$ for the spectral turnover frequency (see previous section and Fig. 6). If we assume that most of the synchrotron energy is radiated near the turnover frequency $v_{m}$, we can estimate the Lorentz factor of the electrons via the relation $v_{m}=1.2 \times$ $10^{-3} B \gamma^{2}$ ( $B$ in $[\mathrm{G}], v_{m}$ in [GHz]) to be $\gamma \sim 2200$, which corresponds to an electron energy of $E_{\mathrm{e}}=\gamma m_{\mathrm{e}} c^{2} \simeq 1.1 \mathrm{GeV}$. First order inverse-Compton scattering would boost this radiation to $v_{\text {ic }} \propto \gamma^{2} v_{m} \sim 10^{17.6} \mathrm{~Hz}$, thus into the X-ray domain. This suggests that the X-ray emission of 3C 279 is dominated by synchrotron-self-Compton emission, while a different component (either Compton scattering of external photons or second-order Compton scattering of synchrotron photons) is needed to reproduce the gamma-ray emission near $\sim 10^{24} \mathrm{~Hz}$, in agreement with our SED modeling (see Sect. 5).

\section{Spectral energy distribution}

The best multiwavelength coverage at IR/optical/UV frequencies was obtained on 15 January 2006, where observations in the near-IR, optical (UBVRI) and UV (UVW1, UVM2, UVW2) frequencies were available within $\sim 1 / 2 \mathrm{~h}$ of UT 05:00. We therefore focus our attention on the simultaneous multiwavelength SED centered on that time. For the X-ray regime, we included the high-quality Chandra spectrum of 17 January 2006 in our SED, which is justified by the moderate X-ray variability during the core campaign. Given the substantial integration time required to obtain spectral information from the INTEGRAL observations, we use the time-averaged spectrum over the entire observation for inclusion in the SED. Justified by the long variability time scales in the radio, data taken within a few days of 15 January 2006 were included to extend the SED into the radio regime. The resulting SED, including radio, IR, optical (WEBT), UV (Swift UVOT) and X-ray (Chandra, INTEGRAL) and soft $\gamma$-ray (INTEGRAL) is displayed in Fig. 8 and compared to the very-low-state SED from the multiwavelength campaign during INTEGRAL AO-1 (June 2003, Collmar et al. 2004). 


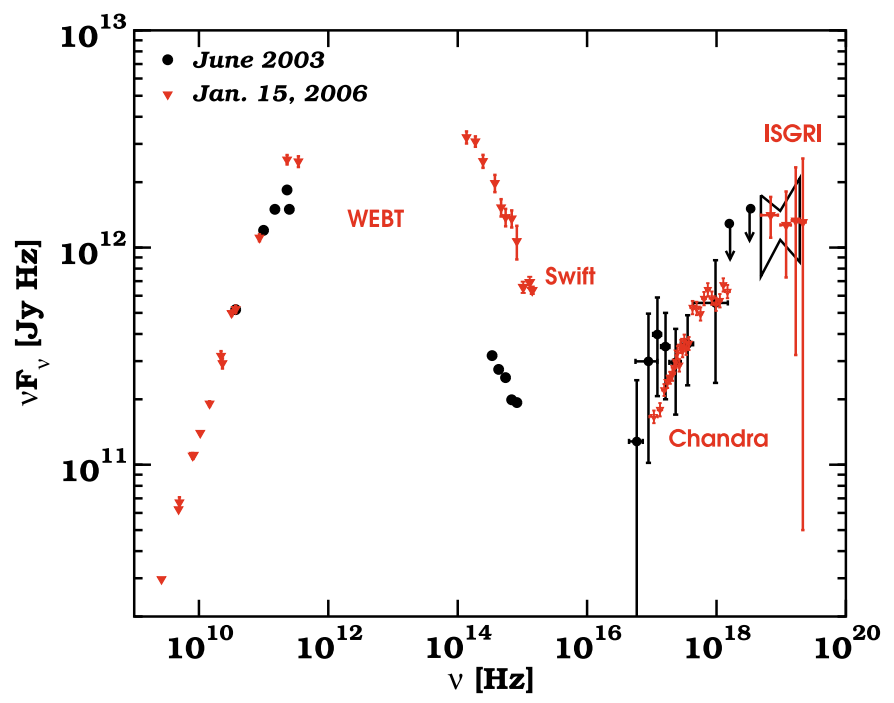

Fig. 8. Spectral energy distribution of $3 \mathrm{C} 279$, centered on 15 January 2006 (red downward triangles). All IR/optical/UV data were taken within $\pm 1 / 2 \mathrm{~h}$ of UT 05:00. Also shown for comparison is the SED from the INTEGRAL AO-1 campaign from June 2003 (Collmar et al. 2004). Despite the huge difference in optical flux, the $X$ - and hard $\mathrm{X}$-ray fluxes are surprisingly similar.

The most remarkable feature of the 15 January 2006 SED is the low X-ray flux, while the optical flux was clearly in an elevated state. The X-ray spectrum is virtually indistinguishable from the historical low-state SED of June 2003, while the optical flux is about a factor of 10 higher. The only slight difference in the X-ray spectral shape is the indication of a steepening toward lower frequencies, possibly indicating a low-energy cutoff of the X-ray spectrum (and hence, the underlying electron distribution, see Sect. 5), for which there was no evidence in the June 2003 SED. A comparison to a further historical SED is shown in Fig. $9^{2}$.

Unfortunately, because the time of this campaign was before the launch of AGILE and Fermi, there was no simultaneous coverage of the critical $\mathrm{MeV}-\mathrm{GeV} \gamma$-ray regime, where the highenergy component of 3C 279 is expected to peak. Therefore, constraints on the peak of the high-energy component can only be based on plausibility arguments in comparison with historical SEDs, an example of which is shown in Fig. 9.

\section{SED modeling}

As demonstrated in Hartman et al. (2001a), a leptonic one-zone jet model, including both SSC and external-Compton scattering as high-energy radiation mechanisms, has been appropriate and very successful for modeling all simultaneous SEDs of 3C 279 collected during the lifetime of EGRET. We therefore choose the same approach to interpret the low X-ray flux states presented in Fig. 8, including the SED from 15 January 2006, the center of the core period of our multiwavelength campaign described here. For this purpose, we use the equilibrium version of the leptonic SSC + EC model of Böttcher \& Chiang (2002).

In this model, a relativistic electron population is assumed to be in an equilibrium state between on-going injection, escape, and radiative cooling in a spherical emission region of

2 The interested reader may contact W. Collmar for an ASCII table of the figure. radius $R$, moving relativistically with bulk Lorentz factor $\Gamma$ along the jet. Electrons are injected with a power-law distribution specified by an injection spectral index $q$ and low- and high-energy cutoffs $\gamma_{1}$ and $\gamma_{2}$, respectively. The radiative cooling is evaluated self-consistently with the radiative output via synchrotron, SSC, and external-Compton emission. Particles are assumed to escape on an energy-independent time scale $t_{\mathrm{esc}} \equiv \eta R / c$, where $\eta>1$ is a free parameter. Particle escape will become relevant for particles with energies below a critical break energy $\gamma_{\mathrm{b}}$ defined through the condition that $t_{\mathrm{esc}}=t_{\mathrm{cool}}$, the radiative cooling time scale. Escaping particles are leaving the emission region in random directions and will therefore primarily end up in a slower sheath that may be present around the fast spine producing the high-energy emission. The magnetic field $B$ is a free parameter of the model, as are the parameters characterizing the external radiation field. For any given set of parameters, the code also evaluates the ratios of co-moving energy densities in the magnetic field, $u_{B}^{\prime}$, and the equilibrium electron distribution, $u_{\mathrm{e}}^{\prime}$, the corresponding kinetic $\left(L_{\mathrm{e}}\right)$ and Poynting flux $\left(L_{B}\right)$ powers, and their ratio, $\epsilon_{B} \equiv u_{B}^{\prime} / u_{\mathrm{e}}^{\prime}=L_{B} / L_{\mathrm{e}}$. For a more detailed description of this equilibrium model see Acciari et al. (2009).

The model fits only the near-infrared to gamma-ray emission, which is believed to be produced at the sub-pc scales of the jet, where the source is still optically thick at radio wavelengths. The optically thick radio emission is believed to be produced on $>$ pc scales. Above $\sim 100 \mathrm{GHz}$ however, the emission comes from sub-parsec scales of a region which is located at or very near the base of the VLBI jet (size of VLBI core at $100 \mathrm{GHz} \leq 10^{18} \mathrm{~cm}$ ). We find that the optical through X-ray SED of 15 January 2006 can be well represented with the following model parameters: $L_{\mathrm{e}}=1.2 \times 10^{45} \mathrm{erg} \mathrm{s}^{-1}, \gamma_{1}=1.5 \times$ $10^{3}, \gamma_{2}=10^{5}, q=3.7, B=0.8 \mathrm{G}, R=3.5 \times 10^{16} \mathrm{~cm}, \Gamma=15$, and an external radiation field energy density $u_{\mathrm{ext}}=2.2 \times$ $10^{-4} \mathrm{erg} \mathrm{cm}^{-3}$. In order to reduce the number of free parameters, we choose the observing angle in a way that the Doppler factor $D \equiv\left(\Gamma\left[1-\beta_{\Gamma} \cos \theta_{\text {obs }}\right]\right)^{-1}=\Gamma$. The magnetic field specified above corresponds to a value slightly below equipartition with $\epsilon_{B}=0.56$. The fit is illustrated by the red solid curve in Fig. 9. While the X-ray spectrum is always produced through SSC emission, the $\gamma$-ray emission is strongly dominated by the EC emission. The model does, indeed, slightly over-predict the soft $\gamma$-ray (ISGRI) flux. However, the ISGRI error bars are large, and our fit can still be considered marginally consistent with the ISGRI spectrum.

Remarkably, the other two low-X-ray states can be modeled with essentially identical parameters, changing only the lowenergy cutoff of the electron distribution. The fits to the P2 and June 2003 spectra shown in Fig. 9 have been generated choosing $\gamma_{1}=750$ for P2 and $\gamma_{1}=550$ for June 2003. As already alluded to before, the higher value of the low-energy cutoff may be responsible for the break in the X-ray spectrum seen in the Chandra data of our January 2006 campaign.

The change in $\gamma_{1}$ affects the peak frequencies of all radiation components as $v_{\mathrm{sy}} \propto \gamma_{1}^{2}, v_{\mathrm{EC}} \propto \gamma_{1}^{2}$, and $v_{\mathrm{SSC}} \propto \gamma_{1}^{4}$, as long as Compton scattering occurs in the Thomson regime near the Compton peaks, which is the case for the parameters adopted here. In addition, increasing $\gamma_{1}$ also has the effect of increasing the synchrotron radiation energy density, hence leading to an increasing SSC contribution in the high-energy SED. In Fig. 9 we included the individual radiation components - synchrotron, SSC, EC (BLR), EC (disk), and the disk emission as dashed, dot-dashed, double-dot-dashed, double-dash-dotted and dotted curves for the 15 January 2006 SED fit. This illustrates the dominant SSC contribution to the soft $\gamma$-ray regime in this fit. In the 


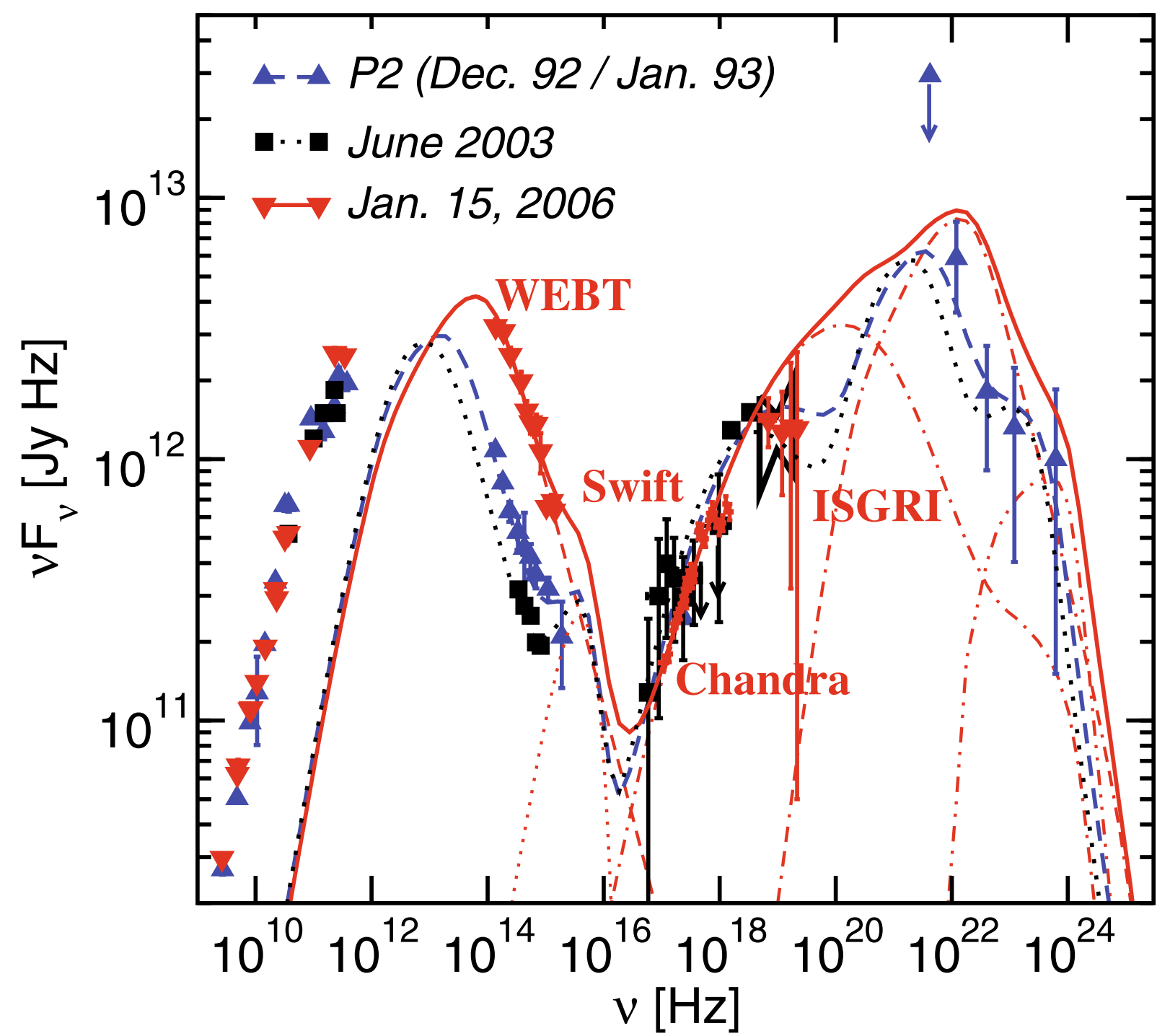

Fig. 9. Fits to the two low-X-ray state SEDs included in Fig. 8, plus a historical low-X-ray state SED from the CGRO era in December 1992/January 1993. The individual model radiation components for the 15 January 2006 SED are shown. See text for details.

states fitted with lower values of $\gamma_{1}$, the SSC component still dominates in the X-ray band, but plays virtually no role in the production of the $\gamma$-ray emission.

In the model calculations for $3 \mathrm{C} 279$, all our cases are in the fast-cooling regime. In the fast-cooling regime, particles will cool below $\gamma_{1}$ to a cutoff energy determined by a balance between radiative cooling and escape. In an internal shock scenario, the value of $\gamma_{1}$ may be related to the relative Lorentz factor of two colliding shells, and the efficiency of transferring sweptup proton energy to accelerated electrons. Because we do not fully understand the details of this transfer process, this latter effect is commonly parameterized through electron acceleration parameters $\epsilon_{\mathrm{e}}$ and $\zeta_{\mathrm{e}}$, both assumed to be on the order of $\sim 10^{-1}$ (e.g., Böttcher \& Dermer 2010). Those parameters are defined so that $\epsilon_{\mathrm{e}}$ is the fraction of swept-up proton energy transferred to relativistic electrons, and $\zeta_{\mathrm{e}}$ the fraction of swept-up electrons that are accelerated to relativistic energies. Specifically, if the electron acceleration process results in an electron spectral index of $q>2$, the low-energy cutoff is given by

$\gamma_{1}=\frac{m_{p}}{m_{\mathrm{e}}} \frac{q-2}{q-1} \frac{\epsilon_{\mathrm{e}}}{\zeta_{\mathrm{e}}}\left(\Gamma_{\text {shock }}-1\right)$, where $\Gamma_{\text {shock }}$ is the Lorentz factor of the (internal) forward or reverse shock resulting from the collision of two shells of relativistic ejecta, measured in the co-moving frame of the shocked material (Böttcher \& Dermer 2010). While $\Gamma_{\text {shock }}$ is a strong function of the relative Lorentz factors of the colliding shells, $\epsilon_{\mathrm{e}}$ and $\zeta_{\mathrm{e}}$ are expected to depend on the efficiency of the generation of turbulent magnetic fields mediating the energy transfer between protons and electrons behind the shock fronts.

In Böttcher et al. (2007), constraints on jet parameters could be derived from the estimated synchrotron peak flux in the 15 January 2006 SED, and a hint for hard lags among the optical $(B V R)$ bands. The synchrotron peak flux was translated into a magnetic-field estimate according to Eq. (4) in Böttcher et al. (2007). Inserting the values of our fit results, $D=15$ and $\epsilon_{B}=0.56$, that estimate becomes

$B \geq B_{\epsilon_{B}}=0.74\left(t_{\mathrm{var}, \mathrm{d}}^{\mathrm{obs}}\right)^{-1} \mathrm{G}$

which perfectly agrees with the adopted value $B=0.8 \mathrm{G}$ in our fit.

Based on evidence for hard lags ( $R$ band leading the $V$ and $B$ band fluxes), Böttcher et al. (2007) developed even more 
parameter constraints based on the assumption that the lags are caused by a slow acceleration process, which needs to be faster than the radiative cooling time scales. This led to the magneticfield estimate in Eq. (6) in Böttcher et al. (2007). Using our fit values as well as the best-fit $B-R$ time lag, $\tau_{B R}=3.75 \mathrm{~d}$, we find

$B \leq B_{\text {acc }}=0.20(1+k)^{-2 / 3} \mathrm{G}$,

where $k$ is the ratio of Compton-to-synchrotron cooling rates (i.e., $k=u_{\mathrm{rad}}^{\prime} / u_{B}^{\prime}$ if Compton scattering occurs in the Thomson regime). This upper limit is substantially lower than our adopted fit value. However, we point out that evidence for hard lags in the WEBT campaign data seems to have been most prominent in the second half of the campaign, where even hints for spectral hysteresis among the optical bands were found. No indication of spectral hysteresis was present during the time corresponding to the 15 January SED fitted in this paper.

\section{Summary and conclusions}

We have observed the prominent FSRQ 3C 279 over a period of about 10 days in an intensive multiwavelength campaign in January 2006. We obtained hard X-ray/soft $\gamma$-ray coverage with INTEGRAL, additional X-ray monitoring observations with Swift XRT, and RXTE, a high-quality $0.3-7 \mathrm{keV}$ X-ray spectrum from Chandra, optical/UV monitoring with Swift/UVOT, and also long-term optical/near-IR/radio monitoring by the WEBT, and the Effelsberg -, UMRAO, IRAM, and ATCA radio telescopes, and the SMA.

Our campaign caught the object initially in a high optical state, followed by a clean, quasi-exponential decline throughout the core campaign period. The X-ray monitoring observations indicate the opposite trend, namely a gradual flux increase. The optical and X-ray fluxes of 3C 279 clearly do not follow a correlated variability trend during our campaign.

The campaign results allowed us to construct a well sampled SED from radio through soft $\gamma$-rays. Comparing this SED to a historical SED from the EGRET era as well as INTEGRAL AO-1 (June 2003), we find that among EGRET P2 (December 1992-January 1993), INTEGRAL AO-1 (June 2003) and our January 2006 campaign, the optical flux level - but not the optical continuum spectral shape - had changed by almost one order of magnitude, while the X-ray flux and spectral shape appear almost unchanged. Our January 2006 SED represents the highest optical flux state among the three SEDs compared here, and is the only SED in which we found evidence for a downward bending in the X-ray spectrum, possibly indicating a low-energy cut-off.

We modeled the SED of January 2006 together with the other two low-X-ray flux SEDs mentioned above, with a onezone leptonic model, including synchrotron, SSC, and externalCompton emission and self-consistently evaluating an equilibrium electron distribution with the radiative output. This model was previously successfully applied to all simultaneous SEDs of 3C 279 collected during the CGRO era. We find that all three low-X-ray SEDs can be well represented with this model. Most remarkably, the optical variability among these three SEDs can be reproduced by changing only the low-energy cutoff of the electron distribution, leaving all other parameters fixed. We caution that this interpretation of the variability is obviously not unique, given the substantial number of parameters entering the model. However, the evidence for a low-energy break in the January 2006 X-ray spectrum lends additional support to the presence of a low-energy cutoff in the electron spectrum at ultrarelativistic energies. This result may have important consequences for the evaluation of the total kinetic power carried by the jet in 3C 279. Given the steep spectral index of the electron spectrum inferred from the steep optical spectrum, most of the kinetic energy in the leptonic component of the sub-pc scale jet is carried by the low-energy end of the electron distribution. Because we lack evidence (e.g., a bulk Compton component, Begelman \& Sikora 1987) for a substantial thermal plasma component in the jet, our results then suggest that the kinetic luminosity in relativistic electrons required for our model fit $\left(L_{\mathrm{e}}=1.2 \times 10^{45} \mathrm{erg} \mathrm{s}^{-1}\right)$ provides a realistic estimate of the total kinetic luminosity in leptons in the relativistic jet.

Acknowledgements. We thank S. Jorstad and A. Marscher for providing VLBI data at $43 \mathrm{GHz}$ and for discussion. We thank the IRAM Granada staff for data from their regular flux monitoring program, and in particular $\mathrm{H}$. Ungerechts and S. Leon for their support. This research is based on observations with the Effelsberg 100-m telescope of the Max-Planck-Institut für Radioastronnomie (Bonn, Germany). This research has made use of data from the University of Michigan Radio Astronomy Observatory (UMRAO), which has been supported by the University of Michigan and the National Science Foundation. This work has made use of observations with the IRAMinterferometer and IRAM 30-m telescope, the Australia Telescope Compact Array, the Very Large Array (NRAO) and the Submillimeter Array (SMA). The Submillimeter Array is a joint project between the Smithsonian Astrophysical Observatory and the Academia Sinica Institute of Astronomy and Astrophysics and is funded by the Smithsonian Institution and the Academia Sinica. M.B. acknowledges support from NASA through INTEGRAL Guest Investigator grant NNX09AI71G. Swift is supported at Penn State by NASA contract NAS500136. D.G. acknowledges support by NASA contract NNX07AH67G. I.A. acknowledges support by the National Science Foundation of the USA and the "Ministerio de Ciencia e Innovación" of Spain through grants AST-0907893, and AYA2007-67627-C03-03, respectively.

\section{References}

Abdo, A. A., Ackermann, M., Ajello, M., et al. 2009, ApJ, 707, 1310 Acciari, V. A., Aliu, E., Aune, T., et al. 2009, ApJ, 707, 612

Agudo, I., Krichbaum, T. P., Ungerechts, H., et al. 2006, A\&A, 456, 117 Aharonian, F. 2000, New Astron., 5, 377

Akujor, C. E., Lüedke, E., Browne, I. W. A., et al. 1994, AAS, 105, 247

Albert, J., Aliu, E., Anderhub, H., et al. 2008, Science, 320, 1752

Angelakis, E., Fuhrmann, L., Marchili, N., Krichbaum, T. P., \& Zensus, J. A. 2008, Mem. Soc. Astron. Ital., 79, 1042

Arnaud, K. A. 1996, Astronomical Data Analysis Software and Systems V, ASP Conf. Ser., 101, 1

Barthelmy, S. D., Barbier, L. M., Cummings, J. R., et al. 2005, Space Sci. Rev., 120,143

Begelman, M. C., \& Sikora, M. 1987, ApJ, 322, 650

Bloom, S. D., \& Marscher, A. P. 1996, ApJ, 461, 657

Böttcher, M. 2007, ApSS, 309, 95

Böttcher, M., \& Chiang, J. 2002, ApJ, 581, 127

Böttcher, M., \& Principe, D. 2009, ApJ, 692, 1374

Böttcher, M., \& Dermer, C. 2010, ApJ, 445, 460

Böttcher, M., Basu, S., Joshi, M., et al. 2007, ApJ, 670, 968

Böttcher, M., Reimer, A., \& Marscher, A. P. 2009, ApJ, 703, 1168

Bottacini, E., Collmar, W., \& Courvoisier, T. J.-L. 2007, Proc. of 6th INTEGRAL Workshop, The Obscured Universe, ed. S. Grebenev, R. Sunyaev, \& C. Winkler, ESA SP-622, 203

Burrows, D. N., David, N., Hill, J. E., et al. 2005, Space Sci. Rev., 120, 165

Cardelli, J. A., Clayton, G. C., \& Mathis, J. S. 1989, ApJ, 345, 245

Chatterjee, R., Jorstad, S. G., Marscher, A. P., et al. 2008, ApJ, 689, 79

Collmar, W., Böttcher, M., Burwitz, V., et al. 2004, Proc. of 5th INTEGRAL Workshop, The INTEGRAL Universe, ed. V. Schönfelder, G. Lichti, \& C. Winkler, ESA SP-552, 555

Collmar, W., Böttcher, M., Krichbaum, T., et al. 2007, Proc. of 6th INTEGRAL Workshop, The Obscured Universe, ed. S. Grebenev, R. Sunyaev, \& C. Winkler, ESA SP-622, 207 
W. Collmar et al.: The multifrequency campaign on 3C 279 in January 2006

Dermer, C. D., \& Schlickeiser, R. 1993, ApJ, 416, 458

Dwek, E., \& Krennrich, F. 2005, ApJ, 618, 657

Fuhrmann, L., Krichbaum, T. P., Witzel, A., et al. 2008, A\&A, 490, 1019

Gehrels, N., Chincarini, G., Giommi, P., et al. 2004, ApJ, 611, 1005

Grupe, D., Komossa, S., Leighly, K. M., \& Page, K. L. 2010, ApJS, 187

Hartman, R. C., Webb, J. R., Marscher, A. P., et al. 1996, ApJ, 461, 698

Hartman, R. C., Bertsch, D. L., Bloom, S. D., et al. 1999, ApJS, 123, 203

Hartman, R. C., Böttcher, M., Aldering, G., et al. 2001a, ApJ, 553, 683

Hartman, R. C., Villata, M., Balonek, T. J., et al. 2001b, ApJ, 558, 583

Hill, J. E., Burrows, D. N., Nousek, J. A., et al. 2004, SPIE, 5165, 217

Jorstad, S. G., Marscher, A. P., Lister, M. L., et al. 2005, AJ, 130, 1418

Larionov, V. M., Jorstad, S. G., Marscher, A. P., et al. 2008, A\&A, 492, 389

Lebrun, F., Leray, J. P., Lavocat, P., et al. 2003, A\&A, 411, L141

Lindfors, E. J., Türler, M., Valtaoja, E., et al. 2006, A\&A, 456, 895

Lund, N., Budtz-Jørgensen, C., Westergaard, N. J., et al. 2003, A\&A, 411, L231

Mannheim, K., \& Biermann, P. L. 1992, A\&A, 253, L21

Marscher, A. P. 2006, Blazar Variability Workshop II: Entering the GLAST Era, 350,155
Marscher, A. P., \& Gear, W. K. 1985, ApJ, 298, 114

Maraschi, L., Celotti, A., \& Ghisellini, G. 1992, ApJ, 397, L5

Maraschi, L., Grandi, P., Urry, C. M., et al. 1994, ApJ, 435, L91

Mas-Hesse, J. M., Giménez, A., Culhane, J. L., et al. 2003, A\&A, 411, L261

Mücke, A., Protheroe, R. J., Engel, R., et al. 2003, Astropart. Phys., 18, 593

Raiteri, C. M., Villata, M., Kadler, M., et al. 2006, A\&A, 459, 731

Roming, P. W. A., Kennedy, T. E., Mason, K. O., et al. 2005, Space Sci. Rev., 120,95

Sikora, M., Begelman, M. C., \& Rees, M. J. 1994, ApJ, 421, 153

Stecker, F. W., \& Scully, S. T. 2009, ApJ, 691, L91

Taylor, G. B., \& Myers, S. T. 2000, VLBA Scientific Memo 26 (Charlottesville: NRAO)

Ubertini, P., Lebrun, F., Di Cocco, G., et al. 2003, A\&A, 411, L131

Ungerechts, H., Kramer, C., Lefloch, B., et al. 1998, ASPC, 144, 149

Urry, C. M., \& Padovani, P. 1995, PASP, 107, 803

Vedrenne, G., Roques, J.-P., Schönfelder, V., et al. 2003, A\&A, 411, L63

Villata, M., Raiteri, C. M., Aller, M. F., et al. 2007, A\&A, 464, L5

Wehrle, A. E., Pian, E., Urry, C. M., et al. 1998, ApJ, 497, 178 